\title{
Utilização de espectrometria de fluorescência de raio $X$ em análises de alimentos
}

\author{
Mariele dos Santos, Francine Novack Victoria
}

https://doi.org/10.4322/mp.978-65-991393-3-8.c4

\section{Resumo}

O alimento é a principal fonte de elementos químicos essenciais, majoritários e traços para animais e seres humanos. A presente revisão reúne pesquisas atuais que usam espectrometria de fluorescência de raios $X$ para análise elementar aplicada em alimentos. Essa análise é fundamental para a avaliação da segurança, já que alguns elementos potencialmente tóxicos, perigosos para a saúde humana podem contaminar os alimentos, entrando na cadeia alimentar a partir do ambiente, do processamento e/ou armazenamento. São necessárias técnicas analíticas rápidas e sensíveis, capazes de detectar elementos maiores e traços na busca de informações da composição multi-elementar e rastreio das fontes de contaminação do produto. A técnica de espectrometria de fluorescência de raio $X$ é uma excelente ferramenta para a análise elementar de alimentos, uma vez que fornece a identificação simultânea de múltiplos elementos em amostras líquidas e sólidas. Diversas matrizes podem ser analisadas obtendo resultados com uma boa precisão e exatidão.

Palavras-chave: alimento, análise elementar, elementos químicos, fluorescência de raio $X$.

\section{Introdução}

Muitos físicos eminentes no final do século XIX acreditavam que a natureza do mundo físico estava substancialmente esclarecida. Entretanto, este paradigma foi rompido já nesta época por uma série de descobertas em um curto intervalo de tempo. Muitas dessas descobertas envolviam inovações científicas provenientes do estudo de radiações e ondas eletromagnéticas. Neste período, ocorreu a descoberta dos raios $X$ em 1895 por Wilhelm Conrad Roentgen, da radioatividade por Henri Becquerel em 1896 e do elétron por Joseph John Thomson em 1897.

Em relação a radiação de raios $X$, quando um raio incide em matéria, vários fenômenos acontecem e o feixe é atenuado, tanto por espalhamento como por absorção. A absorção é mais significativa e é diretamente proporcional ao número atômico (Z) dos elementos químicos, quanto maior o $Z$, maior a absorção de raios $X$. Sendo assim, materiais heterogêneos apresentam diferentes atenuações, as quais dependem do número atômico médio das diferentes áreas irradiadas (RATTI, 2015).

De acordo com Saitovich (1995) entre as descobertas e acontecimentos científicos marcantes que ocorreram nesta época, talvez possa ser dito que a descoberta que possuiu a maior importância para a origem e desenvolvimento das tecnologias baseadas em raios $X$, tenha sido a de Wilhelm Conrad Roentgen a partir do 
experimento com raios catódicos.

As aplicações do raio $X$ foram surgindo com o tempo, tanto baseadas no fenômeno de absorção, como no de espalhamento.

Atualmente as instrumentações que se baseiam em técnicas de raios $X$ deixaram de ser utilizadas apenas para a realização de diagnósticos médicos, essas tecnologias passaram a ser utilizadas na produção de ligas metálicas, tecidos, cerâmicas, entre outros materiais. Além disso, os raios $X$ possuem como característica a capacidade de determinar a porosidade, espessuras de camada, variações de densidade, falhas, inclusões de trincas e outros parâmetros muito utilizados na avaliação do controle de qualidade de diferentes materiais. Hoje são utilizadas no estudo de inúmeras aplicações de diversas áreas da química, biologia, meio ambiente e ciência dos materiais e ciência e tecnologia de alimentos. Podemos dizer, por exemplo, que a técnica de fluorescência de raios X (XRF, sigla em inglês) passou a chamar a atenção de diferentes áreas em virtude do alto potencial que esta técnica possui de fornecer informações de maneira não invasiva, ou seja, por ser uma técnica não destrutiva (NASCIMENTO-DIAS; OLIVEIRA; DOS ANJOS, 2017).

$\mathrm{Na}$ área de ciência e tecnologia de alimentos, estas técnicas são muito utilizadas para o controle de qualidade dos alimentos. A qualidade de um alimento e/ou produto alimentício engloba vários parâmetros, como: tamanho, forma, cor, brilho, consistência, origem, textura, sabor, aspectos nutricionais, métodos de processamento, e, também a contaminação, descoloração, odores indesejáveis e deterioração. Além disso, essas técnicas são importantes para a elaboração de tabelas de composição alimentar, pois a tabela é indispensável no fornecimento de informações para uma dieta balanceada, e a identificação e quantificação dos nutrientes presentes é imprescindível na elaboração da mesma. Outro ponto importante é que existem diferenças nos teores dos nutrientes de uma mesma amostra, devido a fatores de origem da amostra, ambientais e de processamento, sendo assim o controle de qualidade e da composição das amostras é uma ferramenta importante dentro da área de Ciência e Tecnologia de Alimentos.

As técnicas analíticas mais utilizadas para quantificação de nutrientes inorgânicos em alimentos são as que envolvem espectrometria atômica. Geralmente, essas técnicas exigem prévia destruição total ou parcial da matéria orgânica presente na amostra, porém o procedimento oficial de destruição da matéria orgânica, calcinação, requer um tempo relativamente longo para sua execução, além de propiciar perdas dos elementos voláteis, o que tem levado à busca de alternativas mais rápidas

Ao referir-se a todos os perigos que podem tornar os alimentos prejudiciais para a saúde dos consumidores, a segurança dos alimentos é a ferramenta mais utilizada en confiável para garantir a inocuidade e segurança de qualquer alimento ou produto alimentício. Portanto, a definição da qualidade dos alimentos é ampla e envolve a produção, os impactos, os nutrientes e a composição (BORGESE et al., 2015; FAO/WHO, 2003).

Nesse contexto, a composição elementar de alimentos é fundamental para avaliar a presença de elementos nutrientes e a ausência de contaminantes. De La Guardia e Garrigues (2015) propõem a composição elementar dos alimentos baseada em duas classes distintas: elementos traço (faixas de concentrações que variam na ordem de $\mathrm{mg} \cdot \mathrm{kg}^{-1}$ ) e ultratraços (concentrações de microgramas por quilograma $-\mu \mathrm{g} \cdot \mathrm{kg}^{-1}$ ), como pode ser observado na Tabela 1. 
Tabela 1. Classificação dos elementos químicos em traços e ultratraços.

\begin{tabular}{lccc}
\hline Elemento & Essenciais & $\begin{array}{c}\text { Potencialmente } \\
\text { Essenciais }\end{array}$ & $\begin{array}{c}\text { Potencialmente } \\
\text { Tóxicos }\end{array}$ \\
\hline Traço & $\mathrm{F}, \mathrm{I}, \mathrm{Fe}, \mathrm{Si}, \mathrm{Zn}$ & $\begin{array}{c}\mathrm{B}, \mathrm{Ti}, \mathrm{Sb}, \mathrm{As}, \mathrm{Ba}, \\
\mathrm{Ce}, \mathrm{GE}, \mathrm{Rb}, \mathrm{Sr}\end{array}$ & $\begin{array}{c}\mathrm{Al}, \mathrm{As}, \mathrm{Sb}, \mathrm{Cd}, \mathrm{Cr}, \mathrm{Cu}, \\
\mathrm{Pb}, \mathrm{Hg}, \mathrm{Th}, \mathrm{Sn}, \mathrm{U}\end{array}$ \\
\hline Ultratraço & $\begin{array}{c}\mathrm{Cr}, \mathrm{Co}, \mathrm{Cu}, \mathrm{Mn}, \mathrm{Mo}, \\
\mathrm{Ni}, \mathrm{SE}, \mathrm{V}\end{array}$ & & \\
\hline
\end{tabular}

Fonte: REDÍGOLO, 2011.

Os macronutrientes como $\mathrm{Na}, \mathrm{Ca}, \mathrm{Mg}, \mathrm{K}$ e $\mathrm{Cl}$, requerem uma maior quantidade para uma nutrição saudável, enquanto os micronutrientes como $\mathrm{F}, \mathrm{V}, \mathrm{Cr}, \mathrm{Mn}, \mathrm{Fe}, \mathrm{Co}, \mathrm{Cu}$, $\mathrm{Zn}$, Se, Mo e I, são essenciais para nutrição de seres vivos, porém quantias excessivas podem determinar efeitos tóxicos. Por exemplo, $\mathrm{Zn}$ e $\mathrm{Mn}$ são tóxicos quando o nível diário de biodisponibilidade é extrapolado. Outros elementos como Cd, As, $\mathrm{Hg}$ e $\mathrm{Pb}$ são tóxicos mesmo em nível traço e não devem entrar na cadeia alimentar, sob o risco de causar efeitos crônicos a saúde (BAHADIR et al., 2015; DE LA GUARDIA; GARRIGUES, 2015; MONNOT et al., 2016). Indiscutivelmente, a determinação da composição elementar de alimentos é pré-requisito para fazer recomendações a sua ingesta, com destaque no que diz respeito às crianças, gestantes e pessoas com alergias a elementos específicos (ALMEIDA et al., 2008; ANTOINE et al., 2012; MOTAWEI; GOUDA, 2016; SANTOS DA COSTA et al., 2002).

Convém citar a respeito do sentimento comum de que vegetais cultivados em hortas particulares são mais seguros, porém a total falta de controle das áreas de solo privado muitas vezes pode levar a situações de risco exposto (FERRI et al., 2012). O controle, prevenção e monitoramento de elementos tóxicos em alimentos, seja pela contaminação ambiental ou de processamento fazem a análise elementar, cada vez mais necessária na agricultura e na indústria alimentícia. É perceptível que artigos dedicados para a composição elementar de alimentos têm aumentado nos últimos anos e inúmeras fontes de elementos traço foram consideradas (BAHADIR et al., 2015; BILO et al., 2015; BORGESE et al., 2014; DALIPI et al., 2016; DE LA CALLE et al., 2013a; GALLARDO et al., 2016; GEBREKIDAN et al., 2013; SMAGUNOVA; PASHKOVA, 2013).

Com base nesses argumentos, existe uma forte necessidade de ferramentas e técnicas de rastreio com uma ampla faixa de concentração e alta sensibilidade. A espectrometria de massa com plasma indutivamente acoplado (ICP-MS) é a técnica padrão para a análise elementar de alimentos atualmente. A AOAC, nos métodos oficiais de vigilância para monitoramento de rotina, desenvolveu normas e requisitos de desempenho de método para metais pesados em uma variedade de alimentos e bebidas, definindo os valores desejados para o limite de quantificação, repetibilidade, reprodutibilidade, e recuperação. A espectrometria de fluorescência de raio X (XRF) atende esses requisitos para a maioria dos elementos potencialmente tóxicos. É uma das técnicas analíticas quantitativas apropriadas para realizar a análise de elementos principais e traços em alimentos. Muitos estudos demonstram a sua utilização com sucesso para a análise de alimentos (AOAC, 2012; BRUNETTI et al., 2016; GEORGIEVA et al., 2013; JASTRZEBSKA et al., 2003; VIVES et al., 2006; ZARAZUA et al., 2006). 


\section{Sistemas de espectrometria de fluorescência de raio $X$}

Os raios $X$ são radiações eletromagnéticas com frequências superiores à $3 \times 10^{18} \mathrm{~s}^{-1}$ e são produzidos quando elétrons são acelerados em direção a um alvo metálico. $O$ choque do feixe de elétrons (que saem do cátodo com energia de dezenas de $\mathrm{KeV}$ ) com o ânodo (alvo) produz dois tipos de raios $\mathrm{X}$. Um deles constitui o espectro contínuo, e resulta da desaceleração do elétron durante a penetração no ânodo. $O$ outro tipo é o raio $X$ característico do material do ânodo, espectro característico. Assim, cada espectro de raios $X$ é a superposição de um espectro contínuo e de uma série de linhas espectrais características do ânodo, como pode ser visto na Figura 1.

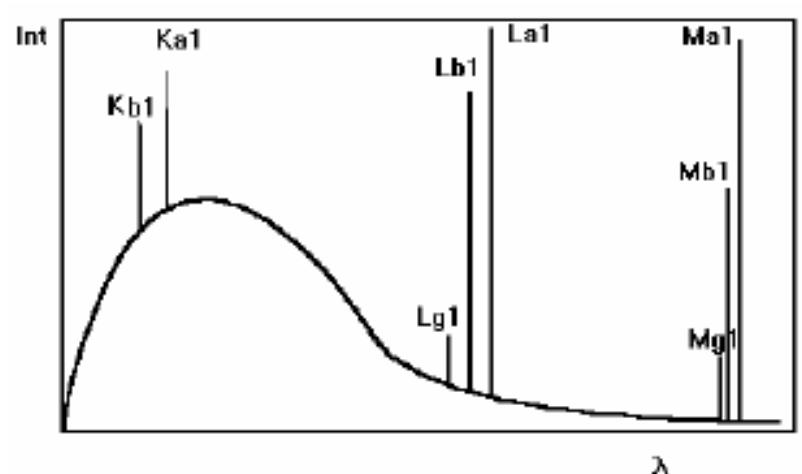

Figura 1. Espectro de comprimentos de onda correspondentes aos raios $X$. Fonte: RATTI, 2015.

O espectro contínuo é simplesmente uma curva de contagens por segundo versus comprimento de onda do raio $\mathrm{X}$, ou seja, Intensidade versus $\mathrm{I}$, como pode ser observado na Figura 2A, neste, todas as curvas têm em comum o fato de que há um comprimento de onda mínimo, abaixo do qual não se observa qualquer raio $X$.

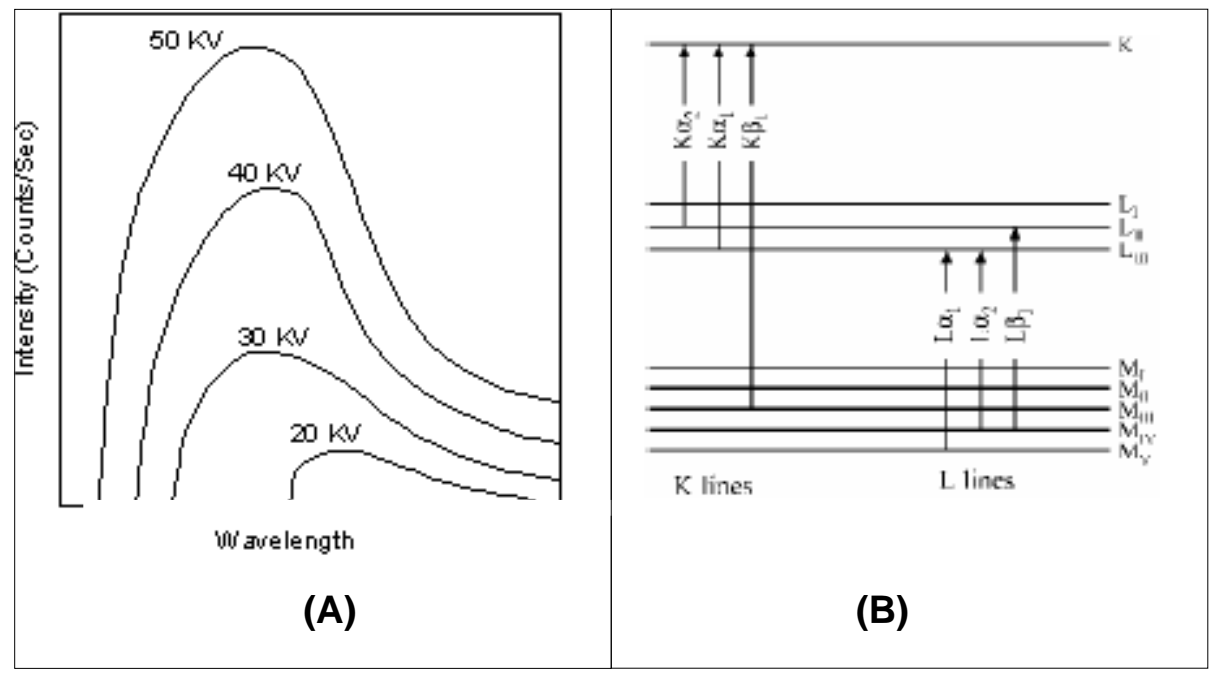

Figura 2. (A) - Espectro contínuo obtido com ânodo de tungstênio (W)

(B) Espectro característico resultante da queda de elétrons alvo Fonte: http://www.tulane.edu/ sanelson/eens211/x-ray.htm.

O espectro característico mostra o rearranjo eletrônico resultante da queda de elétrons-alvo excitados ao nível fundamental. A Figura 2B, apresenta um modelo de espectro característico, neste as linhas de emissão, discretas e superpostas entre si, estas linhas, possuem comprimentos de onda correspondentes aos níveis de $\mathrm{E}$ 
envolvidos e são representadas por letras do alfabeto grego (camadas envolvidas) e números (subcamadas). As linhas traduzem o ponto em que a Energia (E) foi suficiente para remover completamente um dos elétrons-alvo e mostram que outro elétron ocupou o seu lugar, emitindo um fóton de radiação $X$, com comprimento de onda correspondente aos níveis de $\mathrm{E}$ envolvidos e, desta maneira, correspondente ao elemento alvo.

Esse fenômeno pode ser produzido experimentalmente em instrumentos científicos, no interior de um de seus componentes constituídos por um pequeno envoltório de vidro a vácuo chamado de ampola de raios $X$. Dessa maneira, a produção de raios $X$ acontece quando existe a incidência de fótons sobre um anteparo com frequências acima desse valor anteriormente citado e são freados subitamente, passando então para um estado de repouso. Os raios $X$ também podem ser gerados a partir de um alvo material bombardeado por partículas carregadas, como elétrons e prótons, como pode ser observado na Figura 3.
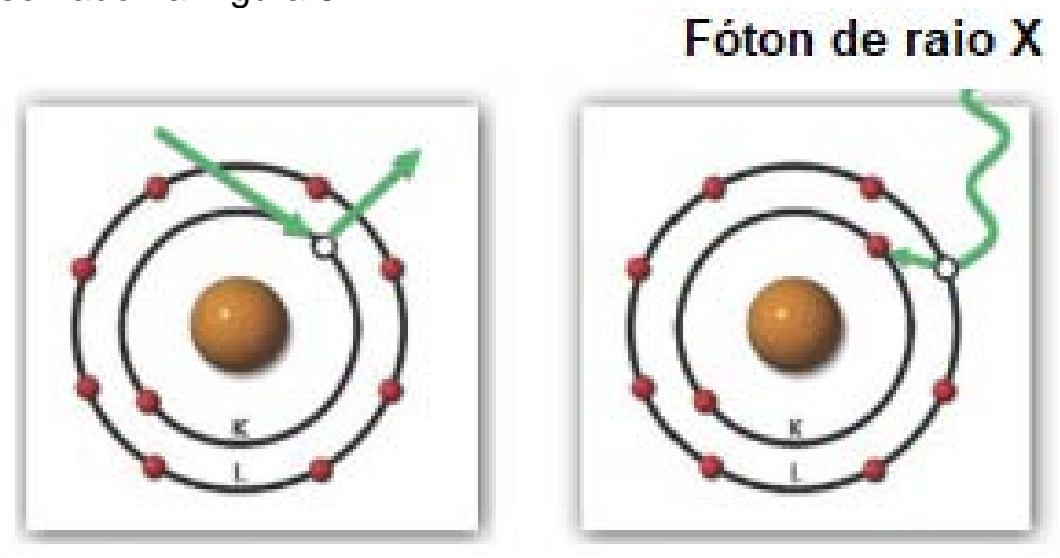

Figura 3. Representação do raio $X$.

Fonte: Manual ARL 9900 Intellipower Series, No AA83654-02, Thermo Fisher Scientific, 2007.

Neste sentido, o fenômeno de produção de raios $X$ pode gerar um espectro de energia (E) duas formas distintas: contínua ou discreta; a forma energética irá depender do tipo de interação que irá ocorrer. O espectro de energia contínua ocorre em função da desaceleração brusca das partículas carregadas e é chamado de efeito brehmsstralung, enquanto a forma discreta ocorre em função de uma reorganização das camadas eletrônicas dos átomos do material do alvo, chamada raios $X$ característicos.

O princípio básico da técnica $X R F$, é o uso de um feixe de raios $X$ para produzir ionização nas camadas internas dos átomos presentes na amostra devido à absorção fotoelétrica. As energias ou comprimentos de onda da emissão dos raios $X$ são então usados para identificar os elementos presentes na amostra. Normalmente, espectrômetros XRF são divididos em duas categorias principais dependendo dos fundamentos do sistema de detecção, são eles: Sistemas de comprimento de onda dispersivo (WDXRF) e sistemas de energia dispersiva (EDXRF e TXRF) (MARGUÍ; ZAWISZA; SITKO, 2014). O arranjo geométrico dos componentes dos sistemas pode ser visto na Figura 4 a seguir. 


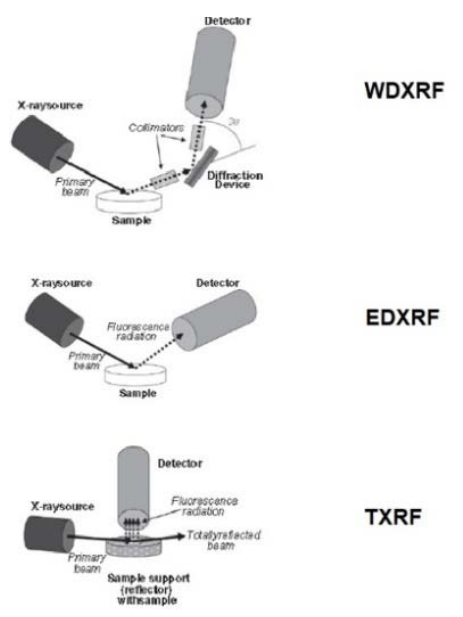

Figura 4. Esquema básico dos sistemas WDXRF, EDXRF e TXRF. Fonte: MARGUÍ; ZAWISZA; SITKO, 2014.

WDXRF emprega difração por um sistema de dispersão para separar os comprimentos de onda característicos emitidos pela amostra. A fonte de raios $X$ irradia a amostra $e$ uma parte da radiação de fluorescência característica da espécie é passada através de um colimador sobre a superfície do dispositivo de difração, onde os comprimentos de onda individuais são difratados para o detector de acordo com a Lei de Bragg. Um goniômetro é usado para manter o ângulo necessário entre o dispositivo de difração e o detector. Devido à potência de alta resolução de cristais e estruturas em camadas múltiplas, fótons correspondentes a linhas características com energias podem ser detectados sem interferir uns nos outros, proporcionando assim uma elevada especificidade na análise. Como interferências espectrais são evitadas, a detecção pode ser realizada utilizando detectores de contador proporcional, com grande eficiência (MARGUÍ; ZAWISZA; SITKO, 2014; SITKO; ZAWISZA, 2005).

Espectrômetros EDXRF, diferente dos sistemas WDXRF, contam com duas unidades básicas: a fonte de excitação e o sistema de detecção. A resolução do seu sistema de energia dispersiva equivale diretamente com a resolução do detector, um detector semicondutor de alta resolução intrínseca. A utilização desse tipo de detector em combinação com um analisador multicanal permite a determinação de todos os raios $X$ emitidos pela amostra ao mesmo tempo, dando uma maior velocidade na aquisição e visualização de dados. Porém, na prática, existe um limite para a taxa de contagem máxima que o detector semicondutor pode manipular. Por esta razão, modificadores de origem, tais como filtros, são geralmente colocados entre a fonte de raio $X$ e a amostra para evitar a saturação do detector. Outra opção é a introdução de um elemento puro ou composto alvo entre a fonte primária e a amostra de tal maneira que somente uma faixa de energia de fótons secundários seja selecionada e incida sobre a amostra, para que ocorra a excitação seletiva em um determinado intervalo de energia. Ambas as configurações, WDXRF e EDXRF, tem sido amplamente utilizadas para a análise de amostras sólidas ou líquidas após o procedimento de préconcentração. A seleção da configuração mais adequada é conforme o propósito. Então, os sistemas de EDXRF são preferidos para informação multi-elementar e WDXRF geralmente é selecionado para uma determinação rápida e precisa de alguns elementos (MARGUÍ; ZAWISZA; SITKO, 2014; SITKO, 2009).

A espectrometria de reflexão total de raios-X (TXRF) é uma variação do sistema EDXRF. Onde o feixe primário atinge a amostra a um ângulo de visão inferior a $0,1^{\circ}$, e não no ângulo típico EDXRF de cerca de $40^{\circ}$ com pouca profundidade de penetração. 
Além disso, devido ao ângulo raso de excitação incidente, o detector pode ser posicionado muito perto da amostra levando a um grande ângulo sólido para a detecção do sinal de fluorescência. Uma melhoria na sensibilidade de sistemas TXRF comparados com espectrômetros EDXRF convencionais são percebidas. TXRF é uma técnica analítica bem estabelecida para a determinação dos elementos em vários tipos de amostras, especialmente amostras líquidas, pós ou micro. Para realizar a análise sob condições de reflexão total, as amostras devem estar como películas finas. Para amostras líquidas, isto é feito por deposição de 5-50 $\mu \mathrm{L}$ de amostra no suporte refletivo com subsequente secagem da gota (BORGESE et al., 2014; MARGUí; ZAWISZA; SITKO, 2014; PESSANHA et al., 2010).

Os sistemas de bancada e TXRF portáteis disponíveis atualmente oferecem procedimentos de operação simples e limite de detecção comparável com dispositivos de grande escala. É possível usar pouca amostra, possibilita calibração linear através da adição de padrão interno, nenhum consumo de gases ou de água de resfriamento e tempo de análise entre 100-1000 s. Infelizmente, pode haver sobreposição de picos, que diminui a precisão da análise quantitativa e o limite no elemento em identificação. As melhores condições de medição são definidas conforme os elementos em questão (BAIRI et al., 2016). A Figura 5 apresenta um modelo de espectro de XRF obtido em um sistema portátil.

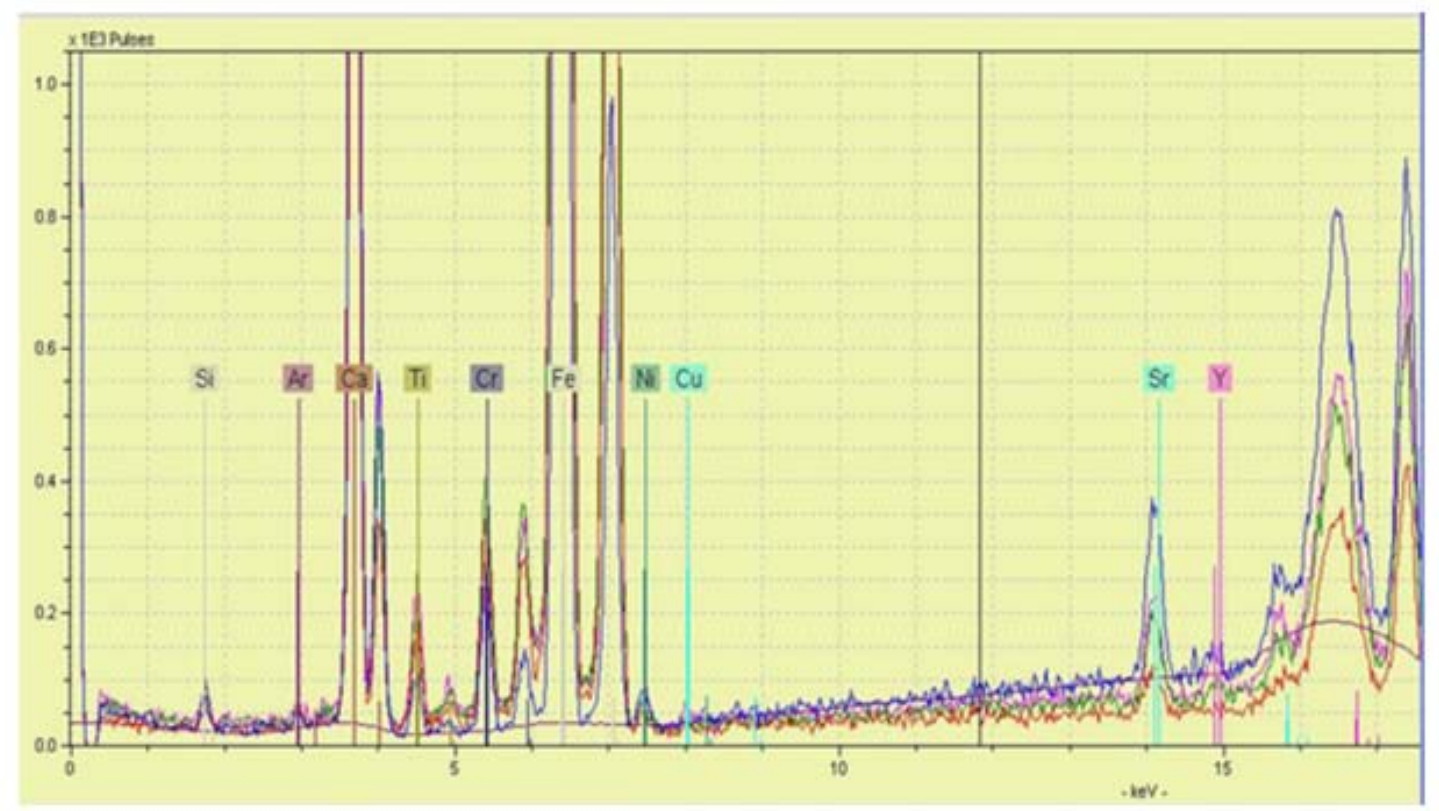

Figura 5. Imagem do espectro de XRF de um modelo portátil. Fonte: NASCIMENTO-DIAS; OLIVEIRA; DOS ANJOS, 2017.

Pré-tratamentos da amostra são geralmente necessários para conseguir uma película fina em matrizes complexas. Os métodos de pré-tratamento da amostra mais comuns são: mineralização, deposição direta da amostra sobre o refletor (suporte) sem ou com tratamento mínimo, suspensões sólidas e extração. Todas essas estratégias permitem realizar análise da maioria dos gêneros alimentícios com boa precisão e exatidão por TXRF. Na análise quantitativa por TXRF é normalmente realizada por calibração com padrão interno. Esse método é simples e fiável, podendo ser empregado para soluções e suspensões. O elemento de padrão interno é normalmente adicionado numa fase inicial de preparação da amostra, mas também pode ser adicionado à amostra depositada sobre o suporte. A confiabilidade da análise quantitativa é 
estimada a partir de precisão e exatidão dos resultados (BAHADIR et al., 2015; DE LA CALLE et al., 2013a; MARGUÍ et al., 2013).

\section{Aplicações}

Encontram-se várias referências que aplicaram a análise multi-elementar quantitativa em bebidas, como por exemplo, água mineral, leite, infusões de chá, café e bebidas alcoólicas. Os elementos principais são geralmente na faixa por cento, enquanto traço e elementos ultra-traço têm concentração em ppm até ppb. O conteúdo dos elementos essenciais é muito diferente em amostras de alimentos, mesmo em matrizes semelhantes (DE LA CALLE et al., 2013b; HASWELL; WALMSLEY, 1998). A seguir serão relatados trabalhos que aplicaram a espectrometria XRF em alimentos visando a composição elementar. Os mesmos estão citados na Tabela 2, a seguir.

Tabela 2. Trabalhos que aplicaram espectrometria de fluorescência de raio $X$ para análise elementar em alimentos.

\begin{tabular}{|c|c|c|}
\hline Referências & Elementos & Amostras \\
\hline \multirow{2}{*}{$\begin{array}{c}\text { HASWELL; WALMSLEY, } \\
1998\end{array}$} & $\begin{array}{l}\mathrm{K}, \mathrm{Ca}, \mathrm{Cr}, \mathrm{Mn}, \mathrm{Fe} \\
\mathrm{Ni}, \mathrm{Cu}, \mathrm{Zn}, \mathrm{Br}\end{array}$ & Café \\
\hline & $\mathrm{Rb}$ & Vinho \\
\hline XIE et al., 1998 & $\begin{array}{l}\mathrm{P}, \mathrm{S}, \mathrm{K}, \mathrm{Ca}, \mathrm{Ti}, \mathrm{Mn}, \\
\mathrm{Fe}, \mathrm{Ni}, \mathrm{Cu}, \mathrm{Zn}, \mathrm{Se} \\
\text { Rb, Sr, Ba, Pb }\end{array}$ & Chá \\
\hline ANJOS et al., 2004 & $\begin{array}{l}\text { V, Fe, } \mathrm{Ni}, \mathrm{Cu}, \mathrm{Zn} \\
\mathrm{Mo}, \mathrm{Pb}\end{array}$ & Vinho \\
\hline ANJOS et al., 2003 & $\begin{array}{c}\mathrm{P}, \mathrm{S}, \mathrm{Cl}, \mathrm{Ca}, \mathrm{Ti}, \mathrm{Cr}, \\
\mathrm{Mn}, \mathrm{Fe}, \mathrm{Ni}, \mathrm{Cu}, \mathrm{Zn}, \\
\mathrm{Rb}, \mathrm{Sr}\end{array}$ & Água \\
\hline GIORGIEVA et al., 2013 & $\begin{array}{c}\mathrm{S}, \mathrm{Cl}, \mathrm{K}, \mathrm{Ca}, \mathrm{Mn}, \\
\mathrm{Fe}, \mathrm{Ni}, \mathrm{Cu}, \mathrm{Zn}, \mathrm{As}, \\
\mathrm{Br}, \mathrm{Rb}, \mathrm{Ba}\end{array}$ & Água \\
\hline BORGESE et al., 2014 & $\begin{array}{c}\mathrm{Cl}, \mathrm{K}, \mathrm{Ca}, \mathrm{Ni}, \mathrm{Sr} \\
\mathrm{Ba}, \mathrm{Br}\end{array}$ & Água \\
\hline MISRA et al., 2009 & $\mathrm{Cl}$ & Água \\
\hline $\begin{array}{c}\text { HATZISTAVROS; } \\
\text { KALLITHRAKAS-KONTOS, } \\
2011\end{array}$ & $\mathrm{ClO}_{4}^{-}$ & Água \\
\hline BAHADIR et al., 2015 & $\mathrm{Cr}$ & Leite \\
\hline $\begin{array}{l}\text { SANTOS DA COSTA et al., } \\
2002\end{array}$ & $\mathrm{Fe}, \mathrm{Cu}, \mathrm{Zn}$ & Maçã \\
\hline
\end{tabular}




\begin{tabular}{ccc}
\hline BAT et al., 2012 & $\mathrm{Mn}$ & Abacaxi \\
\hline PATZ et al., 2013 & $\mathrm{Mn}$ & Cebola \\
\hline ALVAREZ et al., 2003 & $\begin{array}{c}\mathrm{Ca}, \mathrm{K}, \mathrm{Mn}, \mathrm{Fe}, \mathrm{Cu}, \\
\mathrm{Zn}\end{array}$ & Pepino \\
\hline VARGA; ZÁRAY; FODOR, & $\mathrm{Cd}, \mathrm{Ni}, \mathrm{Pb}, \mathrm{Fe}$ & Batata \\
2002 & & Banana \\
& & Chuchu \\
& & Laranja \\
VIVES et al., 2006 & $\mathrm{Al}, \mathrm{P}, \mathrm{K}, \mathrm{Ca}, \mathrm{Ti}, \mathrm{Cr}$, & Alface \\
& $\mathrm{Mn}, \mathrm{Fe}, \mathrm{Co}, \mathrm{Ni}, \mathrm{Cu}$, & Arroz \\
& $\mathrm{Zn}, \mathrm{Mo}, \mathrm{Ba}, \mathrm{Pb}$ & Rúcula \\
& & Feijão \\
\hline
\end{tabular}

Haswell e Walmsley, em 1998, analisaram 11 elementos (Br, Ca, Cr, Cu, Fe, K, Mn, Ni, $\mathrm{Rb}, \mathrm{Sr}$ e $\mathrm{Zn}$ ) por TXRF em amostras de café e vinho de diversos países para que aliado a técnicas multivariadas pudesse determinar suas origens. Seis réplicas foram corridas com um tempo de $500 \mathrm{~s}$ por amostra. As determinações tiveram vanádio (V) como padrão interno. A análise do componente principal demonstrou uma separação entre as amostras de vinho, mas isso não aconteceu para as amostras de café, talvez por ter sido misturado durante o seu processamento a outras amostras de diferentes origens.

Análises multi-elementares em 39 amostras de chá Camellia sinensis (infusões de 0,5 a 85\%) por TXRF demonstraram teores médios entre 0,1 e $30 \mathrm{\mu g} \mathrm{g}^{-1}$, possibilitando o estudo da influência da origem, tipo e qualidade das amostras de chá. O conteúdo de selênio (Se) das amostras de chás cultivados em regiões ricas desse elemento foi elevado $\left(7,5 \mu \mathrm{g} \mathrm{g}^{-1}\right)$, contrastando com a concentração de apenas $0,1 \mu \mathrm{g} \mathrm{g}^{-1}$ de Se nos demais chás examinados (XIE et al., 1998).

Amostras de vinho dos tipos Cabernet Sauvignon, Cabernet Franc, Merlot, Chardonnay, Semillon e Riesling obtidas no mercado local na cidade do Rio de Janeiro foram estudadas no sentido de realizar determinação simultânea de elementos traço. Após digestão ácida, as amostras tiveram seu pH ajustado para 3,0 e sofreram adição de solução pirrolidinaditiocarbamato de amônio (APDC) (1\%). Depois, as amostras foram filtradas a vácuo e consideradas como um filme para serem analisadas pelo método EDXRF, preliminarmente validado. O espectro foi acumulado por $4000 \mathrm{~s}$. Porém, o uso do agente quelante limitou o número de elementos analisados, pois alguns elementos de interesse não pré-concentraram, ficando em níveis abaixo do esperado na literatura. Foi possível determinar $\mathrm{V}, \mathrm{Fe}, \mathrm{Ni}, \mathrm{Cu}, \mathrm{Zn}, \mathrm{Mo}, \mathrm{a} \mathrm{Pb}$ simultaneamente nas amostras de vinho. Os elementos traço no vinho devem ser consequência da composição do solo de origem da videira (ANJOS et al., 2004).

Para determinar simultaneamente a concentração elementar de $\mathrm{P}, \mathrm{S}, \mathrm{Cl}, \mathrm{Ca}, \mathrm{Ti}, \mathrm{Cr}$, $\mathrm{Mn}, \mathrm{Fe}, \mathrm{Ni}, \mathrm{Cu}, \mathrm{Zn}, \mathrm{Rb}$ e $\mathrm{Sr}$ em 27 amostras de vinhos tintos e brancos comerciais através de TXRF foi usado solução de gálio (Ga) como padrão interno. Os teores encontrados foram menores do que os limites estabelecidos pela OIV para vinhos 
(ANJOS et al., 2003).

Várias marcas de água potável engarrafadas (de mesa, de nascente e mineral) da Bulgária foram analisadas por TXRF usando gálio $(\mathrm{Ga})$ como padrão interno. O teor de $\mathrm{S}, \mathrm{Cl}, \mathrm{K}$ e Ca foi no nível mg $\mathrm{L}^{-1}$, enquanto que $\mathrm{Mn}, \mathrm{Fe}, \mathrm{Ni}, \mathrm{Cu}, \mathrm{Zn}, \mathrm{As}, \mathrm{Br}, \mathrm{Rb}$ e $\mathrm{Ba}$ foi inferior ao nível de $\mu \mathrm{g} \mathrm{L}^{-1}$. O conteúdo dos elementos das águas minerais ao longo de um período de armazenamento de aproximadamente 2,5 anos após engarrafamento não apresentaram diferença significativa. Os resultados indicaram que as águas de mesa, de nascente e mineral engarrafadas da Bulgária são um produto de alta qualidade para o mercado búlgaro e europeu das águas potáveis. Também, o método utilizado se mostrou adequado para a análise de elementos traço de rotina das águas potáveis engarrafadas de vários tipos e composição mineral (GEORGIEVA et al., 2013).

Através da técnica de TXRF foi avaliada quantitativamente a composição elementar de amostras de água potável, águas residuais industriais e água dessalinizada MilliQ em 18 laboratórios de 10 países. Ao apresentar e discutir os resultados, a comparação inter-laboratorial teve como objetivo desenvolver diretrizes e uma metodologia padrão para a análise biológica e ambiental, por meio da técnica analítica TXRF. Foram detectados metais e não metais, de alto e baixo peso atômico na evolução dos resultados, mas apenas os elementos $\mathrm{Cl}, \mathrm{K}, \mathrm{Ca}, \mathrm{Ni}$, Sr, $\mathrm{Ba}$ e $\mathrm{Br}$ foram considerados para comparar com os dados obtidos através da utilização de espectrometria de massa com plasma indutivamente acoplado (ICP-MS) por dois diferentes laboratórios em dois países. As principais fontes de erro identificadas foram o tipo de amostra, a preparação da espécie e o procedimento de detecção e quantificação. A fim de reduzir os erros sistemáticos é proposto o uso de massa, no lugar de volume, no preparo da amostra e na adição de padrão interno. O estudo enfatiza a importância da limpeza durante os procedimentos. Os dados e a discussão dos resultados geraram um documento de especificação técnica. O plano futuro é definir uma Internacional Standard Organization (ISO) para análises de água potável por TXRF (BORGESE et al., 2014).

O cloro é adicionado como desinfetante na água de beber para matar bactérias e pode reagir com metais presentes na água para formar cloretos. Acima do limite, o $\mathrm{Cl}$ tornase perigoso para a saúde humana. Seu limite estabelecido pela OMS na água potável é de $5 \mathrm{mg} \mathrm{L}^{-1}$. Um estudo determinou $\mathrm{Cl}$ em água potável por TXRF através da adição de um excesso conhecido de $\mathrm{AgNO}_{3}$ para precipitar todo $\mathrm{Cl}$ contido como $\mathrm{AgCl}$. Essa metodologia pode ser aplicada em soluções que contêm mais de $5 \mu \mathrm{g} \mathrm{m}^{-1}$ de $\mathrm{Cl}$. $\mathrm{O}$ teor de $\mathrm{Cl}$ nas amostras analisadas foi de 1,6 a $56 \mu \mathrm{g} \mathrm{m} \mathrm{m}^{-1}$. Foi usado solução de cádmio (Cd) como padrão interno e o tempo de análise do espectro foi de $1500 \mathrm{~s}$ (MISRA et al., 2009; WHO, 2011).

Um método para a determinação seletiva de ânions perclorato $\left(\mathrm{ClO}_{4}{ }^{2-}\right)$ em nível traço após a remoção do cloreto foi estudado, alcançando boa seletividade com o uso de membranas. Ânions perclorato foram concentrados sobre membranas aniônicas preparadas na superfície de refletores de quartzo para TXRF. Várias substâncias de complexação foram usadas nas membranas. Os refletores foram imersos em soluções de água contendo nanogramas por mililitro $\left(\mathrm{ng} \mathrm{mL}^{-1}\right)$ ou partes por bilhão (ppb) de perclorato. Após esse passo, os refletores foram retirados da solução e analisados por TXRF. Foram examinados os efeitos de vários parâmetros experimentais e a possibilidade de discriminação entre os ânions cloreto e o perclorato. Os limites mínimos de detecção foram inferiores a $1 \mathrm{ng} \mathrm{mL}^{-1}$ e concentrações entre 1 e $50 \mathrm{ng} \mathrm{mL}^{-}$ ${ }^{1}$ foram alcançadas. O método é aplicável para a análise de perclorato em amostras de água (HATZISTAVROS; KALLITHRAKAS-KONTOS, 2011). 
Determinações de cromo (Cr) hexavalente (IV) em água mineral e água da torneira por TXRF detectaram o teor mínimo de $3 \mu \mathrm{g} \mathrm{L}^{-1}$ realizando pré-tratamento da amostra por microextração com nanotubos de carbono de multicamadas modificadas (MWCNT) e solução de ítrio $(Y)$ como padrão interno. Esse valor é cerca de 20 vezes menor do que o teor máximo de cromo hexavalente admissível em águas de beber, de acordo com a Organização Mundial de Saúde (OMS). Conforme os autores, entre os parâmetros que afetam o processo de extração estão o pH e volume da amostra aquosa, a quantidade de MWCNT e o tempo de extração, enquanto que na análise por TXRF são muito importantes o volume de padrão interno, o volume da suspensão depositada sobre o refletor, modo de secagem, e os parâmetros instrumentais (BAHADIR et al., 2015; WHO, 2011).

A composição elementar do leite humano tem atraído o interesse de pesquisadores, uma vez que representa a mais completa nutrição para o recém-nascido e crianças até dois anos de idade. Uma pesquisa mediu a concentração de $\mathrm{Fe}$, Cu e Zn em amostras de colostro de 50 mulheres brasileiras. O pré-tratamento da amostra consistiu em digestão ácida, adição de padrão interno $(\mathrm{Ga})$ e secagem a vácuo no suporte. Cada suporte foi medido por $300 \mathrm{~s}$ no TXRF. Os seguintes valores foram obtidos: $1,72 \mathrm{mg} \mathrm{L}^{-1}$ de Fe; $0,54 \mathrm{mg} \mathrm{L}^{-1}$ de $\mathrm{Cu} ; 6.97 \mathrm{mg} \mathrm{L}^{-1}$ de $\mathrm{Zn}$. As concentrações dos elementos determinados foram comparáveis com os valores encontrados nas referências. Concluindo que TXRF é uma técnica adequada para avaliar elementos inorgânicos no colostro, fornecendo uma análise multi-elementar em uma única medida, podendo ser otimizada, a fim de avaliar uma vasta gama de elementos traço no leite humano (SANTOS DA COSTA et al., 2002).

Em vegetais, frutas, ervas e temperos também são encontradas investigações recentes através de procedimentos de preparo de amostra e aplicação de XRF. A maioria utiliza digestão ácida, análise direta, extração ultrassônica ou digestão assistida por micro-ondas na preparação. A seguir algumas pesquisas recentes são reportadas.

Suco de maçã adicionado de padrão de $\mathrm{Ga}$, através de análise direta, após a homogeneização em banho ultrassônico por uma hora, foi submetido à TXRF para determinação multi-elementar em maçãs da Eslovênia. As frações médias em massa dos elementos seguiram a ordem: $\mathrm{K}>\mathrm{P}>\mathrm{Ca}>\mathrm{S}>\mathrm{Cl}>\mathrm{Rb}>\mathrm{Zn}>\mathrm{Mn}$. Maçãs Gala tiveram as maiores frações médias de massa de $P$ e $K$ e as menores de $M n, Z n$ e Rb. Maçãs Goldrush apresentaram as maiores frações médias de massa de S, Cl e Zn e a menor fração média massa de K. Foram observadas as maiores frações médias de massa de Ca, Mn e Rb em maçãs Topaz, Gloster e Golden Delicious, respectivamente. Através de análise discriminante linear, os teores de $\mathrm{Mn}, \mathrm{Zn}$ e $\mathrm{Rb}$ apontaram maior contribuição com a origem botânica, enquanto os teores de Rb e S contribuíram para distinguir as regiões geográficas de origem das maçãs. As indicações geográficas são cada vez mais usadas como ferramentas de marketing, pois podem agregar valor econômico aos produtos agrícolas por transmitir uma identidade cultural através dos produtos (BAT et al., 2012).

O elemento manganês $(\mathrm{Mn})$ é essencial para a nutrição humana e é encontrado no abacaxi em concentrações elevadas. Com base nisso, o teor de manganês do abacaxi, do suco, concentrado e purê de abacaxi foram analisados por espectrometria de fluorescência de raios $X$ com reflexão total para análise elementar. As amostras foram trituradas, filtradas e homogeneizadas num banho ultrassônico durante $5 \mathrm{~min}$. Selênio (Se) foi adicionado como padrão interno numa concentração final de $5 \mathrm{mg} \mathrm{kg}^{-1}$. Duas gotas de $5 \mu \mathrm{L}$ das amostras preparadas foram colocadas sobre o suporte de quartzo e secas a $50{ }^{\circ} \mathrm{C}$ para submeter a TXRF. As concentrações de manganês 
variaram bastante em frutas inteiras, contudo, a casca apresentou maior concentração comparada a polpa da fruta. Os processados de abacaxi, como purês, tem o menor teor de manganês com uma mediana de $4,9 \mathrm{mg} \mathrm{kg}^{-1}$ em comparação com sucos de abacaxi, $10,5 \mathrm{mg} \mathrm{kg}^{-1}$ e concentrados $15,5 \mathrm{mg} \mathrm{kg}^{-1}$. Dependendo da concentração do produto, a ingesta diária tolerável de manganês é praticamente atingida com apenas com um copo de $200 \mathrm{~mL}$ de suco de abacaxi (PATZ et al., 2013).

A cebola (Allium cepa L) é um dos cultivares mais importantes do mundo. Sua caracterização química é importante para a melhoria da eficiência de produção visto que a qualidade e estado de conservação dos produtos agrícolas são associados à sua composição química. A técnica de fluorescência de reflexão total de raios $X$ (TXRF) foi aplicada com sucesso para a determinação simultânea dos elementos Ca, $\mathrm{K}, \mathrm{Mn}, \mathrm{Fe}$, Cu e $\mathrm{Zn}$ em dois cultivares de cebola, com foco para o desenvolvimento de um procedimento de preparo da amostra que envolve a extração ácida dos analitos a partir de amostras cruas por meio de um banho de ultrassom. As técnicas de espectrometria de absorção atômica com chama (FAAS) e espectrometria de absorção atômica com chama e injeção em fluxo foram empregadas para fins de comparação entre os teores dos elementos. Os níveis de $\mathrm{K}, \mathrm{Mn}$ e $\mathrm{Zn}$ foram significativamente superiores no cultivar 438 Granex, enquanto que os níveis de $\mathrm{Ca}$, $\mathrm{Fe}$ e $\mathrm{Cu}$ foram significativamente maiores no cultivar Yellow Granex PRR 502. O método de preparação proposto foi o mais confiável, simples, rápido e usa menos produtos químicos e vidrarias, quando comparado com os métodos clássicos de calcinação por via úmida ou seca, proporcionando qualidade analítica similar. A técnica TXRF permitiu quantificar os elementos $\mathrm{Cu}, \mathrm{Fe}$ e $\mathrm{Mn}$, não realizado pela técnica FIAAS e em comparação com a técnica FAAS permitiu a quantificação de Cu (ALVAREZ et al., 2003).

A distribuição de $\mathrm{Cd}, \mathrm{Ni}, \mathrm{Pb}$ e Fe entre o citoplasma e a parede celular de raízes e folhas da planta do pepino foi determinado por espectrometria de reflexão total de fluorescência de raios-X, usando gálio (Ga) como padrão interno, após o preparo da amostra por digestão assistida por micro-ondas. O suporte com amostra seca foi irradiado por $500 \mathrm{~s}$. Nas raízes a maior parte da $\mathrm{Pb}$ foi encontrado na parede celular, enquanto $\mathrm{Ni}$ e $\mathrm{Cd}$ no citoplasma. Nas folhas, o citoplasma continha de $50-60 \%$ do teor de $\mathrm{Pb}, 60-80 \%$ de Fe e praticamente todo o teor $\mathrm{Cd}$, enquanto, cerca de $30-40 \%$ de $\mathrm{Ni}$ estava na parede celular. Uma vantagem da técnica TXRF utilizada é a possibilidade de analisar pequena quantidade de amostra (VARGA; ZÁRAY; FODOR, 2002).

Os resultados de uma pesquisa indicaram que algumas espécies analisadas estavam contaminadas por $\mathrm{Cr}$, $\mathrm{Cu}, \mathrm{Zn}$ e $\mathrm{Pb}$, com concentrações muito mais elevadas do que os valores permitidos pela legislação brasileira. $O$ estudo utilizou fluorescência de raios $X$ por reflexão total para a determinação simultânea e multi-elementar (Al, P, K, Ca, Ti, $\mathrm{Cr}, \mathrm{Mn}, \mathrm{Fe}, \mathrm{Co}, \mathrm{Ni}, \mathrm{Cu}, \mathrm{Zn}, \mathrm{Br}, \mathrm{Mo}, \mathrm{Ba}$ e $\mathrm{Pb}$ ) de alimentos disponíveis para a população em estabelecimentos comerciais, a fim de avaliar os riscos de contaminação por esses produtos. Os limites máximos permitidos de acordo com a ANVISA para Cr, Ni, Cu, Zn e Pb são: 0,1, 5, 30, 50 e $0,5 \mathrm{mg} \mathrm{kg}^{-1}$, respectivamente e para Al $5 \mu \mathrm{g} \mathrm{g}^{-1}$. Os alimentos analisados e os elementos que estavam acima do limite permitido nas amostras foram: arroz, $\mathrm{Cr}$, $\mathrm{Cu}$ e $\mathrm{Pb}$; feijão, $\mathrm{Pb}$ e $\mathrm{Al}$; batata, $\mathrm{Cr}, \mathrm{Pb}$ e Al; banana, $\mathrm{Cu}$ e $\mathrm{Pb}$; chuchu, $\mathrm{Pb}$; laranja, $\mathrm{Pb}$ e $\mathrm{Al}$; alface, $\mathrm{Cr}$; rúcula, $\mathrm{Cr}$, $\mathrm{Cu}$ e $\mathrm{Pb}$ (VIVES et al., 2006).

\section{Considerações finais}

As técnicas de espectrometria de fluorescência de raio $X$ é uma técnica analítica não destrutiva, com baixo custo de análise, baixo consumo de reagentes e vidraria, gera 
pouco ou nenhum resíduo, sendo uma boa solução para as análises de rotina. Uma das vantagens da TXRF em relação à WDXRF e EDXRF é que a técnica não requer métodos para correção do efeito matriz, pois são utilizadas quantidades muito pequenas das amostras, as quais são depositadas em um suporte, formando um filme fino. Graças a fina espessura da amostra e alta energia dos raios X utilizados na excitação, não há ocorrência do efeito de absorção e, por isso, não é necessária a correção para o efeito matriz.

A análise elementar dos produtos alimentares tem significativa importância, já que a toxicidade dos elementos inorgânicos pode ocorrer em níveis pouco acima da ocorrência natural. Logo, o consumo de alimentos com uma alta concentração desses elementos pode causar intoxicação aguda ou crônica. A técnica de espectrometria de fluorescência de raio $X$ oferece uma rápida e confiável análise quantitativa. $A$ sua utilização na determinação de elementos majoritários, essenciais e traços em bebidas e alimentos, trazendo avanços tecnológicos na instrumentação e a combinação do aprimoramento do pré-tratamento da amostra foram apresentados nesta revisão.

Será necessário desenvolver diretrizes para procedimentos experimentais, padrões de processamento de dados e de amostras referência. Assim como, iniciativas interlaboratoriais para avaliar as abordagens analíticas e a reprodutibilidade dos protocolos. Isso porque, apesar do desenvolvimento de pesquisas e publicações de procedimentos, protocolos e resultados confiáveis, a análise quantitativa por espectrometria de fluorescência de raio $X$ é ainda limitada a investigação, principalmente devido à falta de métodos padrão reconhecidos.

\section{Referências}

ALMEIDA, A. A. et al. Trace elements in human milk: Correlation with blood levels, inter-element correlations and changes in concentration during the first month of lactation. Journal of Trace Elements in Medicine and Biology, v. 22, n. 3, p. 196205, 2008. https://doi.org/10.1016/j.jtemb.2008.03.007.

ALVAREZ, J. et al. Determination of calcium, potassium, manganese, iron, copper and zinc levels in representative samples of two onion cultivars using total reflection X-ray fluorescence and ultrasound extraction procedure. Spectrochimica Acta Part B: Atomic Spectroscopy, v. 58, n. 12, p. 2183-2189, 2003. https://doi.org/10.1016/S0584-8547(03)00222-2.

ANJOS, M. J. et al. Trace elements determination in red and white wines using totalreflection X-ray fluorescence. Spectrochimica Acta Part B, v. 58, p. 2227-2232, 2003. https://doi.org/10.1016/j.sab.2003.07.004.

ANJOS, M. J. et al. EDXRF analysis of red and white wines from Brazil. X-Ray Spectrometry, v. 33, n. 6, p. 407-409, 2004. https://doi.org/10.1002/xrs.737.

ANTOINE, J. M. R. et al. Dietary intake of minerals and trace elements in rice on the Jamaican market. Journal of Food Composition and Analysis, v. 26, n. 1, p. 111121, 2012. https://doi.org/10.1016/j.jfca.2012.01.003.

AOAC. Appendix F: Guidelines for Standard Method Performance Requirements. AOAC Official Methods of Analysis, p. 1-17, 2012.

BAHADIR, Z. et al. Determination of trace amounts of hexavalent chromium in drinking waters by dispersive microsolid-phase extraction using modified multiwalled carbon 
nanotubes combined with total reflection X-ray fluorescence spectrometry. Spectrochimica Acta Part B: Atomic Spectroscopy, v. 107, p. 170-177, 2015. http://dx.doi.org/10.1016/j.sab.2015.03.010.

BAIRI, V. G. et al. Portable X-ray fluorescence spectroscopy as a rapid screening technique for analysis of $\mathrm{TiO} 2$ and $\mathrm{ZnO}$ in sunscreens. Spectrochimica Acta Part B: $\begin{array}{llllll}\text { Atomic Spectroscopy, } & \text { v. 116, p. 21-27, } 2016 .\end{array}$ https://doi.org/10.1016/j.sab.2015.11.008.

BAT, K. B. et al. Characterization of Slovenian apples with respect to their botanical and geographical origin and agricultural production practice. Food Technology and Biotechnology, v. 50, n. 1, p. 107-116, 2012.

BILO, F. et al. Evaluation of heavy metals contamination from environment to food matrix by TXRF: The case of rice and rice husk. Journal of Chemistry, v. 2015, p. 112, 2015. https://doi.org/10.1155/2015/274340.

BORGESE, L. et al. First Total Reflection X-Ray Fluorescence round-robin test of water samples: Preliminary results. Spectrochimica Acta Part B: Atomic Spectroscopy, v. 101, p. 6-14, 2014. https://doi.org/10.1016/j.sab.2014.06.024.

BORGESE, L. et al. Total reflection X-ray fluorescence as a tool for food screening. Spectrochimica Acta - Part B Atomic Spectroscopy, v. 113, p. 1-15, 2015. https://doi.org/10.1016/j.sab.2015.08.001.

BRUNETTI, A. et al. X-ray fluorescence spectroscopy (XRF) and Monte Carlo characterization of a unique nuragic artifact (Sardinia, Italy). Spectrochimica Acta Part B: Atomic Spectroscopy, v. 121, p. 18-21, 2016. https://doi.org/10.1016/j.sab.2016.04.007.

DALIPI, R. et al. Analytical performance of benchtop total reflection X-ray fluorescence (TXRF) instrumentation for multielemental analysis of wine samples. Spectrochimica Acta Part B: Atomic Spectroscopy, v. 120, p. 37-43, 2016. https://doi.org/10.1016/j.sab.2016.04.001.

DE LA CALLE, I. et al. Sample pretreatment strategies for total reflection X-ray fluorescence analysis: A tutorial review. Spectrochimica Acta - Part B Atomic Spectroscopy, v. 90, p. 23-54, 2013a. https://doi.org/10.1016/j.sab.2013.10.001.

DE LA CALLE, I. et al. Fast method for multielemental analysis of plants and discrimination according to the anatomical part by total reflection X-ray fluorescence spectrometry. Food Chemistry, v. 138, n. 1, p. 234-241, 2013b. https://doi.org/10.1016/j.foodchem.2012.09.105.

DE LA GUARDIA, M.; GARRIGUES, S. Handbook of Minerals Elements in Food. [s.l.] Chichester: Willey Blackwell, 2015. 772p.

FAO/WHO. Assuring Food Safety and Quality: Guidelines for strenghtening national food control systems. Rome: Joint FAO/WHO Publication, 2003.

FERRI, R. et al. Heavy metals in soil and salad in the proximity of historical ferroalloy emission. Journal of Environmental Protection, v. 3, p. 374-385, 2012. https://doi.org/10.4236/jep.2012.35047.

GALLARDO, H. et al. Bromine and bromide content in soils: Analytical approach from 
total reflection X-ray fluorescence spectrometry. Chemosphere 156, v. 156, p. 294301, 2016. https://doi.org/10.1016/j.chemosphere.2016.04.136.

GEBREKIDAN, A. et al. Toxicological assessment of heavy metals accumulated in vegetables and fruits grown in Ginfel river near Sheba Tannery, Tigray, Northern Ethiopia. Ecotoxicology and Environmental Safety, v. 95, p. 171-178, 2013. https://doi.org/10.1016/j.ecoenv.2013.05.035.

GEORGIEVA, R. et al. Total reflection X-ray fluorescence analysis of trace elements in Bulgarian bottled mineral waters of low and high mineral content. International Journal of Environmental Analytical Chemistry, v. 93, n. 10, p. 1043-1051, 2013. https://doi.org/10.1080/03067319.2013.775277.

HASWELL, S. J.; WALMSLEY, A. D. Multivariate data visualisation methods based on multi-elemental analysis of wines and coffees using total reflection $\mathrm{X}$-ray fluorescence analysis. Journal of Analytical Atomic Spectrometry, v. 13, n. 2, p. 131-134, 1998. https://doi.org/10.1039/A705317G.

HATZISTAVROS, V. S.; KALLITHRAKAS-KONTOS, N. G. Determination of trace perchlorate concentrations by anion-selective membranes and total reflection $\mathrm{X}$-ray fluorescence analysis. Analytical Chemistry, v. 83, n. 9, p. 3386-3391, 2011. https://doi.org/10.1021/ac103295a.

JASTRZEBSKA, A. et al. Determination of phosphorus in food samples by X-ray fluorescence spectrometry and standard spectrophotometric method. Food Chemistry, v. 83, p. 463-467, 2003. https://doi.org/10.1016/S0308-8146(03)00225-5.

MARGUÍ, E. et al. Liquid phase microextraction strategies combined with total reflection X-ray spectrometry for the determination of low amounts of inorganic antimony species in waters. Analytica Chimica Acta, v. 786, p. 8-15, 2013. https://doi.org/10.1016/j.aca.2013.05.006.

MARGUÍ, E.; ZAWISZA, B.; SITKO, R. Trace and ultratrace analysis of liquid samples by X-ray fluorescence spectrometry. TrAC - Trends in Analytical Chemistry, v. 53, p. 73-83, 2014. https://doi.org/10.1016/j.trac.2013.09.009.

MISRA, N. L. et al. A novel approach for chlorine determination in acidic medium by total reflection x-ray fluorescence. X-Ray Spectrometry, v. 38, n. 3, p. 182-185, 2009. https://doi.org/10.1002/xrs.1139.

MONNOT, A. D. et al. Risks associated with arsenic exposure resulting from the consumption of California wines sold in the United States. Food Chemistry, v. 211, p. 107-113, 2016. https://doi.org/10.1016/j.foodchem.2016.05.013.

MOTAWEI, S. M.; GOUDA, H. E. Screening of blood levels of mercury, cadmium, and copper in pregnant women in Dakahlia, Egypt: New attention to an old problem. Biological Trace Element Research, v. 171, n. 2, p. 308-314, 2016. https://doi.org/10.1007/s12011-015-0525-y.

NASCIMENTO-DIAS, B.L.; OLIVEIRA, D.F.; DOS ANJOS, M.J. A utilização e a relevância multidisciplinar da fluorescência de raios $X$. Revista Brasileira de Ensino de Física, v. 39, n 4, p. 4308, 2017. https://doi.org/10.1590/1806-9126-rbef-2017-0089. 
PATZ, C.-D. et al. Manganese screening of pineapple by total-reflection X-ray fluorescence (TXRF) spectroscopy. Deutsche Lebensmittel-Rundschau, v. 109, n. 6, p. 315-319, 2013.

PESSANHA, S. et al. Quantitative determination on heavy metals in different stages of wine production by Total Reflection X-ray Fluorescence and Energy Dispersive X-ray Fluorescence: Comparison on two vineyards. Spectrochimica Acta Part B: Atomic $\begin{array}{lllllll}\text { Spectroscopy, } & \text { v. } 65, \quad \text { n. } & 6, & \text { p. }\end{array}$ https://doi.org/10.1016/J.SAB.2010.04.003.

\section{RATTI, G. Técnicas de análise química de compostos inorgânicos. 2015}

REDÍGOLO, M.M. Determinação de elementos químicos inorgânicos em amostras de sangue total humano e de animais de experimentação (hamster dourado e cavalo de raça crioula) pela técnica de fluorescência de raio X (EDXRF). Dissertação (Instituto de Pesquisas Nucleares e Energéticas). Universidade de São Paulo, 2011.

SAITOVITCH, H. Cem anos da descoberta dos raios-X: Caracterização estrutural de sóidos. Revista Brasileira de Ensino de Física, v. 17, n. 3,1995.

SANTOS DA COSTA, R. S. et al. Trace Elements Content of Colostrum Milk in Brazil. Journal of Food Composition and Analysis, v. 15, n. 1, p. 27-33, p.203, 2002. https://doi.org/10.1006/jfca.2001.1038.

SITKO, R. Quantitative X-ray fluorescence analysis of samples of less than "infinite thickness": Difficulties and possibilities. Spectrochimica Acta - Part B Atomic Spectroscopy, v. 64, p. 1161-1172, 2009. https://doi.org/10.1016/j.sab.2009.09.005.

SITKO, R.; ZAWISZA, B. Calibration of wavelength-dispersive X-ray spectrometer for standardless analysis. Spectrochimica Acta - Part B Atomic Spectroscopy, v. 60, p. 95-100, 2005. https://doi.org/10.1016/j.sab.2004.11.004.

SMAGUNOVA, A. N.; PASHKOVA, G. V. Choice of optimal conditions for X-ray fluorescence analysis of milk products with varying fat content. X-Ray Spectrometry, v. 42 , n. 6 , p. 546-551, 2013. https://doi.org/10.1002/xrs.2519.

VARGA, A.; ZÁRAY, G.; FODOR, F. Determination of element distribution between the symplasm and apoplasm of cucumber plant parts by total reflection X-ray fluorescence spectrometry. Journal of Inorganic Biochemistry, v. 89, n. 1, p. 149-154, 2002. https://doi.org/10.1016/S0162-0134(01)00397-X.

VIVES, A. E. S. et al. Synchrotron radiation total reflection X-ray fluorescence (SRTXRF) for evaluation of food contamination. Journal of Radioanalytical and Nuclear Chemistry, v. 270, n. 1, p. 147-153, 2006. https://doi.org/10.1007/s10967-006-0322-3.

WHO. Guidelines for drinking-water quality. Fourth ed. Geneva: World Health Organization, 2011.

XIE, M. et al. Multielement analysis of Chinese tea (Camellia sinensis) by totalreflection X-ray fluorescence. Zeitschrift für Lebensmitteluntersuchung und Forschung A, v. 207, n. 1, p. 31-38, 1998. https://doi.org/10.1007/s002170050291.

ZARAZUA, G. et al. Analysis of total and dissolved heavy metals in surface water of a Mexican polluted river by total reflection X-ray fluorescence spectrometry. Spectrochimica Acta Part B: Atomic Spectroscopy, v. 61, n. 10, p. 1180-1184, 
2006. https://doi.org/10.1016/j.sab.2006.06.010.

\section{Autores}

Mariele dos Santos ${ }^{1}$, Francine Novack Victoria ${ }^{2, *}$

1. Curso de Pós-Graduação Lato Sensu em Ciência dos Alimentos, Centro de Ciências Químicas, Farmacêuticas e de Alimentos, Universidade Federal de Pelotas, Campus Capão do Leão, Caixa Postal 354, CEP. 96010-900, Pelotas, RS, Brasil; Instituto Federal Farroupilha Campus São Vicente do Sul, Diretoria de Produção, Rua 20 de Setembro, 2616, CEP 97420-000, São Vicente do Sul, RS, Brasil.

2. Centro de Ciências Químicas, Farmacêuticas e de Alimentos, Universidade Federal de Pelotas, Campus Capão do Leão, Caixa Postal 354, CEP. 96010-900, Pelotas, RS, Brasil.

*Autor para correspondência: francinevictoria@yahoo.com.br 